\title{
VARIATION AT THE INSERTION OF PALMARIS LONGUS TENDON: A CASE REPORT
}

Padmaja Vasi ${ }^{1}$, Seema Madan ${ }^{2}$

\section{HOW TO CITE THIS ARTICLE:}

Padmaja Vasi, Seema Madan. "Variation at the Insertion of Palmaris Longus Tendon: A Case Report". Journal of Evolution of Medical and Dental Sciences 2014; Vol. 3, Issue 23, June 09; Page: 6478-6480,

DOI: $10.14260 /$ jemds/2014/2767

\begin{abstract}
Palamaris Longus is a superficial flexor of forearm which springs from the median epicondyle of humerus and is inserted into the palmar aponeurosis. It is often used in tendon grafts for replacement of long flexors of the fingers due to its length and diameter. Hence removal of palmaris longus tendon may not produce functional deformities. In recent times the palmaris longus tendon has gained importance in reconstructive surgeries thus it is essential to know its variations. A case of a variant pattern of insertion of palmaris longus tendon was observed during routine dissection classes for under graduate students in Department of Anatomy. It was noted that the tendon merged with thenar muscles and few fibres of the tendon were seen attached to palmar aponeurosis. Knowledge of variations of the palmaris longus tendon could be of great importance to surgeons, especially to those operating on the hand.
\end{abstract}

KEYWORDS: Palmaris Longus, Thenar muscle, Palmar aponeurosis.

INTRODUCTION: Palmaris Longus tendon is slender fusiform muscle which springs from medial epicondyle of humerus by a common tendon, from adjacent intra muscular septa and deep fascia. It converges into a long tendon which passes anterior (superficial) to flexor retinaculum. A few fibers leave the tendon and interweave with the transverse fibers of flexor retinaculum. As the tendon crosses the retinaculum it broadens out to become a flat sheet which splits longitudinally to send bundles of ligamentous fibers to digital rays.

These bundles occupy the triangular area in the mid palm which is called as palmar aponeurosis. Palmaris Longus has wide insertion to skin, fascia, in the digital palm and webs. Variations of the Palmaris longus have been frequently observed. ${ }^{1}$ Palmaris Longus tenses the palmar aponeurosis thus stabilizes the palmar skin in grasping the object. The muscle is frequently absent. ${ }^{2}$

CASE HISTORY: During the routine dissection of embalmed cadavers for teaching first year M.B.B.S. students, a variation was noticed at the insertion of palmaris longus tendon in the left upper extremity of a 60years old male cadaver. Palmaris Longus tendon was identified on the superficial stratum of flexor retinaculum. It was observed that median nerve was projecting laterally from behind the palmaris longus tendon. Majority of the tendinous fibers were inserted into the thenar eminence and few medial fibers were inserted into palmar aponeurosis. In the same cadaver palmaris longus was normal in right upper extremity as shown in fig. 1.

DISCUSSION: Variations in the insertion of Palmaris Longus tendon has been widely described by various authors. The muscle may get inserted into the fascia of the fore arm, thenar fascia, hypothenar fascia, pisiform bone or scaphoid bone.3,4 Numerous anomalous origin, course, insertion of palmaris longus have been observed by various authors. ${ }^{5}$ Insertion is also highly variant and 
muscle may be attached to fascia of forearm, tendon of flexor carpi ulnaris. ${ }^{6}$ Abnormal insertion of palmaris longus, deep to flexor retinaculum produces carpal tunnel syndrome. Compression of median nerve by distal belly of palmaris longus and also three headed Palmaris longus muscle was observed and found to be the cause of compartment syndrome. ${ }^{7}$ Functionally, palmaris longus is a weak flexor, but it has much importance in applied aspects.

It is utilized for elective reconstructive surgeries and autografts because of its length and diameter. It is also used in lip augmentation ${ }^{8}$ and ptosis correction, ${ }^{9,10}$ in management of facial palsy. ${ }^{11}$ Insertion of the tendon into abductor pollicis brevis was observed by Valeria et al in 2007.12

The present case study revealed a rare variation of insertion of palmaris longus tendon, which was predominantly merging with the thenar muscle mainly to flexor pollicis brevis and few fibres of the tendon were inserting into palmar aponeurosis. The median nerve was projecting laterally from behind the palmaris longus tendon which is a normal finding.

\section{REFERENCES:}

1. Peter L.Williams, Grey's Anatomy 38th Edition, 1999 Churchill Livingstone. Page -846.

2. Romanes G J, Cunningham's manual of Practical Anatomy Vol-1 15th Edition, 2005, page - 87.

3. Durgun B, Celik HH, Atasever A, Yilmaz E, Hayran M. An unusual insertion of the palmaris longus muscle. Kaibogaku Zasshi. 1993 Feb; 68 (1):104-6. Pub Med PMID: 8517112.

4. Dr. Mobin N, Dr. Saraswati G. Anatomical variations of Palmaris Longus muscle. Anatomica Karnataka 2010; 4 (2): 74-77.

5. Pai MM, Prabhu LV, Nayak SR, Madhyastha S, Vadgaonkar R, Krishnamurthy A, Kumar A. The palmaris longus muscle: its anatomic variations and functional morphology. Rom J Morphol Embryol. 2008; 49: 215-217.

6. Lemon M, Belcher H J, An anomalous flexor carpi ulnaris. J Hand Surgery (Br) 2002, 22(2): 1947.

7. Natsis K, Levva S, Totlis T, Anastasopoulos N, Paraskevas G. Three-headed reversed palmaris longus muscle and its clinical significance. Ann Anat. 2007; 189: 97-101. 


\section{CASE REPORT}

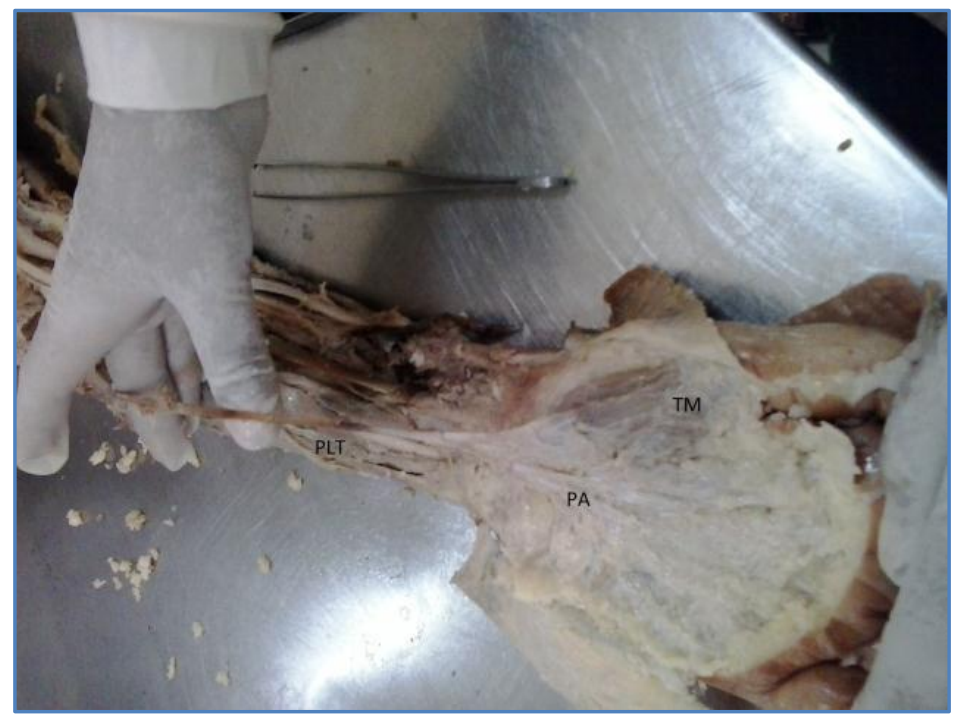

\section{Fig. 1: A rare variation at the insertion of Palmaris Longus Tendon}

PLT: Palmaris Longus Tendon,

TM: Thenar Muscle,

PA: Palmar aponeurosis.

\section{AUTHORS:}

1. Padmaja Vasi

2. Seema Madan

\section{PARTICULARS OF CONTRIBUTORS:}

1. Assistant Professor, Department of Anatomy, Gandhi Medical College, Secunderabad, Andhra Pradesh, India.

2. Professor and HOD, Department of Anatomy, Gandhi Medical College, Secunderabad, Andhra Pradesh, India.

\section{NAME ADDRESS EMAIL ID OF THE}

\section{CORRESPONDING AUTHOR:}

Dr. Padmaja Vasi,

Flat No. 302, Tulip Pearl Apartments,

Road No. 1, Alkapuri Ramakrishnapuram (PO),

Hyderabad-500035.

E-mail: padmaja.v1968@gmail.com

Date of Submission: 05/05/2014.

Date of Peer Review: 06/05/2014.

Date of Acceptance: 27/05/2014.

Date of Publishing: 07/06/2014. 